\title{
Modelo estratégico para optimizar la productividad de la empresa M\&D Catering
}

\section{Strategic model to optimize productivity of M\&D Catering Company}

\author{
Gustavo La Mota Terranova ${ }^{1}$ \\ glamota@uteg.edu.ec \\ https://orcid.org/0000-0003-2783-6644 \\ Pedro Iglesias Mora ${ }^{2}$ \\ piglesias@uteg.edu.ec \\ https://orcid.org/0000-0002-6379-2872 \\ Jorge Zea Maridueña ${ }^{3}$ \\ contador@uteg.edu.ec \\ https://orcid.org/0000-0001-7523-6403
}

Recibido: 07/12/2020, Aceptado: 26/03/2021

\begin{abstract}
RESUMEN
El presente estudio busca a través de un análisis de la cadena de valor de la empresa, determinar oportunidades de mejora reflejadas en el diseño de una planificación estratégica para la empresa, que permita mejorar la productividad de la empresa medida en la eficacia y eficiencia organizacional que se tenga con los clientes y en el manejo de los recursos, como objetivo principal se busca proponer una planificación estratégica para el año 2020 enfocada en la optimización de la productividad de la empresa, mediante el análisis de los procesos y elementos de la cadena de valor para proponer estrategias y actividades que optimicen la productividad. Para la recolección de la información se utilizó la entrevista a profundidad hacia los gerentes responsables de cada una de las dimensiones de la cadena de valor de la empresa y la observación directa para la verificación del cumplimiento de los procesos clave. Las oportunidades de mejora encontradas en el estudio permitieron establecer un modelo estratégico aplicando balanced scorecard para la planificación estratégica de la empresa MyD Catering en el periodo 2020, en el que se resaltan las principales estrategias en sus diferentes dimensiones para aprovechar las oportunidades de mejora.
\end{abstract}

Palabras clave: planificación, cadena de valor, análisis FODA, productividad, retención clientes

\section{ABSTRACT}

This study seeks, through an analysis of the company's value chain, to determine opportunities for improvement reflected in the design of strategic planning for the company, which allows improving the productivity of the company measured in the effectiveness

\footnotetext{
${ }^{1}$ Universidad Tecnológica Empresarial de Guayaquil, Ecuador

2 Universidad Tecnológica Empresarial de Guayaquil, Ecuador

${ }^{3}$ Universidad Tecnológica Empresarial de Guayaquil, Ecuador

Revista científica Ciencia y Tecnología Vol 21 No 30 págs. 86-101

http://cienciaytecnologia.uteg.edu.ec
} 


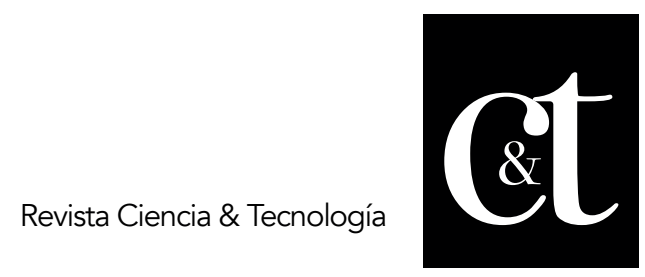

No. 30, 30 de abril de 2021

ISSN impreso: 1390 - 6321

ISSN online: 2661 - 6734

and organizational efficiency that The main objective is to propose a strategic planning for the year 2020 focused on optimizing the productivity of the company, through the analysis of the processes and elements of the value chain. to propose strategies and activities that optimize productivity. To collect the information, an in-depth interview was used with the managers responsible for each of the dimensions of the company's value chain and direct observation to verify compliance with the key processes. The improvement opportunities found in the study made it possible to establish a strategic model applying a balanced scorecard for the strategic planning of the myd catering company in the 2020 period, in which the main strategies in their different dimensions are highlighted to take advantage of the improvement opportunities.

Keywords: planning, value chain, swot analysis, productivity, customer retention.

\section{Introducción}

La globalización de la economía, el desarrollo de nuevas tecnologías de información, la innovación en el sector industrial ha generado una dinámica en las empresas que ha hecho que se transforme la manera tradicional de dirección y gestión, lo cual ha convertido a la cadena de valor en un instrumento de vital importancia para las empresas que pretendan mejorar su eficiencia operativa y activar nuevas estructuras de cooperación con otras empresas. (Sablon Cossío , Medina Leon, Acevedo Suarez, Acevedo Urquiaga, \& Lopez Joy, 2013)

De acuerdo con el estudio del modelo de gestión en la industria del catering enfocada en PYMES del Ecuador, estas empresas en el país cuentan con varias debilidades y amenazas en común como la precarización laboral del talento humano, inadecuada gestión de los recursos, falta de efectividad en los procesos, endeudamiento excesivo, entre otras deficiencias en común (Carrión Erazo, Zula Cujano, \& Castillo Guilcapi, 2016).

En los años recientes la industria el catering ha tenido más espacio en el sector empresarial de PYMES al abrir nuevos campos de negocio diferentes a los convencionales que eran eventos y servicio de catering para buques y aviones, y ha desarrollado nuevas líneas como la elaboración de almuerzos corporativos, escolares, con nuevas propuestas enfocadas hacia los diferentes clientes (Carrión Erazo, Zula Cujano, \& Castillo Guilcapi, 2016).

La cadena de valor de una empresa ya se ha utilizado como una estrategia de desarrollo y crecimiento para el sector de alimentos de acuerdo con Iglesias Mora (2015), en el cual resalta que la habilidad para poder controlar la operación en las actividades primarias y de apoyo en la cadena de valor, constituyen un flujo de vital importancia para el crecimiento de las empresas agroalimentarias.

Esta investigación se realiza con el propósito de aportar al conocimiento existente sobre la aplicación de la cadena de valor, como herramienta para diseñar una planificación estratégica que evidencie mejoras en la productividad de las empresas de servicios de catering. 
Los resultados son presentados en una propuesta, que podría ser considerada y aplicable a otras empresas de la industria de catering en el Ecuador.

En las PYMES, donde no se tiene una planificación estratégica en su modelo de gestión, se evidencian muchos casos en los que las empresas cierran o no prosperan, esto conlleva a la necesidad que aplicar un modelo de planificación estratégica que influya favorablemente en la permanencia y competitividad de las PYMES (Iglesias Mora, 2015).

Esta investigación se realiza por la necesidad de mejorar los niveles de productividad de las Empresa MYD Catering para lograr el cumplimiento de la misión y la visión, a través de una planificación estratégica que utilice la cadena de valor para determinar sus principales insumos.

Utilizando un análisis cualitativo de los elementos de la cadena de valor de la empresa se conocerá la situación actual de los procesos clave de la empresa y a partir de ella proponer un conjunto de estrategias, actividades y tareas enfocadas en el cumplimiento de la visión que tiene la empresa de crecer en el mercado.

La observación directa de las actividades y procesos de la compañía permitirá establecer las explicaciones relacionadas al planteamiento del presente estudio.

La elaboración y aplicación del análisis de la cadena de valor de la empresa y el diseño de la planificación estratégica enfocada en las oportunidades de mejora detectadas, podrá servir como insumo para futuras aplicaciones o estudios en otras empresas PYMES en la industria del catering en el Ecuador.

\section{Empresas de Catering}

Se conoce como catering a los servicios de alimentación empresarial, institucional o colectiva que ofrece al mercado una cantidad de alimentos (comidas y bebidas) en eventos, fiestas u otras actividades empresariales o sociales, en la actualidad estos servicios se han ido desarrollando hacia diferentes líneas de negocio de las cuales se derivan las siguientes clasificaciones de servicios de catering de acuerdo a su naturaleza (Castillo, Zula, \& Carrión, 2016):

Catering Aéreo: Empresas dedicadas a proveer servicios de alimentación para las aerolíneas, enfocadas en el consumo de pasajeros y personal de tripulación que van dentro de los aviones.

Catering de Ferrocarriles: Empresas dedicadas a proveer alimentos para consumo en trenes y ferrocarriles, este tipo de catering no existe en el Ecuador debido no contar con este tipo de transportación de forma masiva.

Catering de buques: Empresas dedicadas a abastecer a alimentación a la tripulación y pasajeros de buques, barcos y todo tipo de medio de transporte marítimo. 
Catering Industrial: Servicio de alimentación enfocado a empresas de industria generalmente alejadas de la ciudad de acuerdo con los requerimientos del cliente, estos pueden ser desayunos, almuerzos y meriendas.

Catering Social: Servicio de alimentación en lugares fuera del ámbito empresarial generalmente en ubicaciones 0 situaciones requeridas específicamente por el cliente (eventos, cumpleaños, bodas, etc.)

Catering Temático: Una derivación del catering social en el cual se centra varios elementos culturales y se presenta una propuesta de servicio enfocada específicamente en un conjunto especifico de características.

Catering Corporativo: Servicio de alimentación dedicado a empresas donde las mismas contratan de manera específica alimentación diaria o en ocasiones especiales para los colaboradores.

\section{La cadena de valor}

Las empresas PYME generalmente no utilizan la planificación por cuatro razones: tiempo, dificultad para empezar, falta de experiencia y falta de confianza. Sin embargo, las empresas que si lo realizan han demostrado contar con muchas ventajas para la realización de la planificación como lograr mejores procesos, adaptación a los cambios, mejor crecimiento traducido en una alta eficacia y eficiencia en la organización. (Saavedra Garcia \& Espíndola Armenta, 2016)

La definición de cadena de valor indica que es una herramienta por la cual, a través de varias etapas y procesos, agrega valor a los distintos clientes y otros grupos de interés. De manera que se sostiene la ventaja competitiva y la derivación de estrategias de la empresa. (Porter, 1982)

El valor es la cantidad de personas dispuestas a comprar los productos que la empresa oferta, una empresa es rentable si el valor es mayor a los costos relacionados del producto. (Olmedo, Olmedo, \& Plazaola)

\section{Elementos primarios de la cadena de valor}

Las actividades primarias son las relacionadas a la creación del producto/servicio, así como sus procesos clave de venta y distribución a los clientes se dividen de acuerdo con las siguientes categorías:

\section{Logística de entrada}

Incluye los procesos de almacenamiento, y recepción de la materia prima para elaborar el producto o servicio.

Es considerada de vital importancia ya que es un insumo primordial que afecta a todo el resto de la cadena de valor, pues sin los insumos para elaborar los productos, no se puede analizar las variables posteriores.

\section{Operaciones}

Las operaciones comprenden los procesos que transforman la materia prima, en productos y servicios para los clientes, tienen una importancia clave en el desarrollo de la empresa pues desde este elemento se desarrolla el cumplimiento de necesidades y satisfacción de los clientes. 


\section{Logística de Salida}

Incluye los procesos de procesamiento de los pedidos, manejo de inventarios de la empresa, y gestión de los canales de distribución para llevar el producto hacia los clientes.

\section{Marketing y Ventas}

Estos procesos están directamente relacionados con las actividades que la empresa realiza para organizar su estrategia comercial enfocada en las ventas, incluye el análisis de todos los elementos que la empresa considera en su estrategia de marketing: Producto, Precio, Distribución y Comunicación (Publicidad, Promoción, Fuerza de Ventas, Etc.).

\section{Servicio Posventa}

Una vez vendido el producto o contratado el servicio, es necesario mantener la relación con los clientes, esto implica el seguimiento que se hace a los clientes para construir relaciones largas y duraderas, incluye también los procesos de evaluación y satisfacción de los clientes.

\section{Actividades de apoyo de la Cadena de Valor}

Las actividades de apoyo o de soporte, son aquellas que ayudan a mantener a las actividades primarias proporcionando elementos, generales que coadyuvan a las actividades primarias en la creación de valor para la empresa.

\section{Infraestructura de la Empresa}

La infraestructura de la empresa comprende las actividades diarias de operación de la compañía, incluye la planificación, el financiamiento y la estructura de relación con los inversores y grupos de interés externos.

\section{Gestión de Recursos Humanos}

En este grupo de procesos de analizan el manejo de los subsistemas de recursos humanos que apoyan a toda la organización: (Reclutamiento y Selección, Capacitación, Remuneraciones, Desarrollo Organizacional, Evaluación, etc.)

\section{Desarrollo de la Tecnología}

Todas las actividades y procesos enfocados en la innovación de los productos y servicios de la empresa, incluye sistemas de información gerencial, sistemas de administración y control de operaciones y otros procesos que evidencien innovación en la forma de realizar las operaciones en la empresa.

\section{Compras}

Comprende los procesos de adquisición de materia y suministros para el funcionamiento y operación de la empresa, en todas sus dimensiones, tanto en actividades primarias como en actividades de apoyo.

\section{Margen}

El margen que detalla la cadena de valor es la diferencia entre el valor total generado por la empresa, y los costos ocurridos de la gestión de actividades primarias y secundarias de la empresa (Quintero \& Sánchez, 2006). 
La cadena de valor comprende todas las fases y etapas básicas del desarrollo de la relación comercial con los clientes, pues va desde los proveedores en la logística de entrada hasta la relación con los clientes en el servicio posventa, por lo cual una vez realizado un análisis de la cadena de valor y detectadas las oportunidades de mejora para determinar ventaja competitiva, se debe diseñar estrategias que permitan el cumplimiento de la misión de la empresa (Quintero \& Sanchez, 2006).

\section{Planeación estratégica}

La planeación estratégica es un proceso a través del cual la empresa plantea sus objetivos y las actividades a realizar para alcanzar un cumplimiento deseado, siguiendo un proceso formal para fijar los principales propósitos y estrategias, en resumen, se refiere al proceso de preparación necesario de la empresa para el cumplimiento de la misión y la visión.

La planeación estratégica permite a la empresa aproximarse a la construcción de un futuro, generalmente visto como un proceso para cumplir los propósitos y las diferentes estrategias que se utilizarán para el cumplimiento de los objetivos (Ramirez Rojas, 2017).

Los principales objetivos de la planeación estratégica son conseguir un enfoque sistemático, fijar objetivos para la empresa, ejecutar estrategias para el cumplimiento de dichos objetivos y orientar a toda la organización para el cumplimiento de actividades y propósitos.

La gestión de las estrategias parte de las habilidades de la dirección, pero es de responsabilidad de cada miembro de la empresa que ejerce una función gerencia o de dirección en la misma (Romo Morales \& Marquez de Leon, 2014).

La planificación se vuelve una práctica constante en la que se relaciona las estrategias (actividades), con los fines, propósitos y resultados que se planteen en la organización, como resultado de los análisis internos y externos realizados, mismos que se reflejan en el mejoramiento de la toma de decisiones (Sánchez Quintero, 2003).

La importancia de la planeación estratégica influye en los demás elementos básicos de la administración, ya que sin una planificación no habría como organizar correctamente los recursos, la dirección tendría poca o nula efectividad en los resultados y sería muy complicado el control de la operación y los cumplimientos, además de volver difícil el tomar acciones correctivas para corregir novedades suscitadas en el desarrollo de la organización (Palacios Acero).

El cuadro de mando integral o balanced scorecard, es una herramienta de gestión estratégica usada principalmente para:

- Alinear las actividades de la empresa con la misión y la visión.

- Mejorar las comunicaciones internas y externas.

- Controlar el rendimiento y el cumplimiento de la organización. 
El balanced scorecard, es una herramienta de gestión que permite realizar los controles enfocados en las actividades claves del negocio, nace de la necesidad de los directivos para tener una visión completa de la operación de la empresa y su rendimiento (Sánchez Vásquez, Velez Elorza, \& Araujo Pinzón, 2016). Está compuesto por cuatro perspectivas que permiten identificar con mayor facilidad las causas y efectos de las actividades y los objetivos.

\section{Perspectiva financiera}

La perspectiva financiera involucra la visión de las empresas, principalmente mide la creación de valor en la empresa, dentro de esta perspectiva se pueden identificar dos grandes variables en muchas organizaciones:

- Crecimiento y diversificación de los ingresos

- Mejora en la productividad y reducción de costos de la empresa.

Los objetivos financieros sirven de foco principal para las demás perspectivas que se proceden a analizar en el balanced scorecard.

\section{Perspectiva de clientes}

En la perspectiva del cliente, las empresas se enfocan en identificar diferentes segmentos de mercado y clientes, para determinar las propuestas de valor que se vayan a utilizar para cumplir los objetivos y retener los clientes.

La perspectiva de clientes debe contener un grupo de indicadores centrales a la operación y a los objetivos de esta perspectiva que son los siguientes:

- Cuota de Mercado

- Incremento de clientes

- Adquisición de clientes

- Satisfacción de clientes

- Rentabilidad de los clientes

\section{Perspectiva interna}

La perspectiva interna es aquella en la cual se analizan y enfocan los procesos más críticos a la hora de conseguir los objetivos, el proceso de derivar indicadores para la perspectiva interna de la empresa representa una de las distinciones más importantes del cuadro de mando integral.

Cada organización tiene un conjunto de procesos enfocados en la creación de valor y producir resultados de rentabilidad y productividad. Estos deben enfocarse primordialmente en tres procesos claves de la organización:

- Innovación

- Operaciones

- Servicio Posventa

\section{Perspectiva de innovación y aprendizaje}

La perspectiva de innovación y aprendizaje es aquella encargada de desarrollar indicadores enfocados en el crecimiento continuo y el aprendizaje de la organización, dentro de la cual hay tres variables importantes de analizar (Kaplan \& Norton, 2000): 


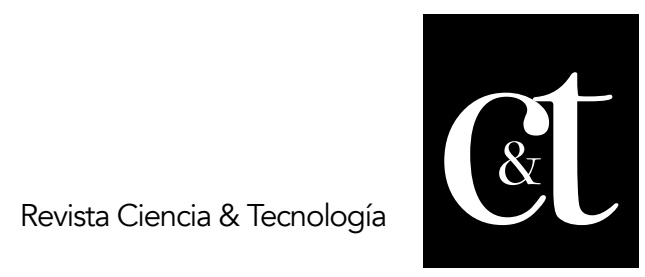

No. 30,30 de abril de 2021

ISSN impreso: 1390 - 6321

ISSN online: 2661 - 6734

- Las capacidades de los empleados

- Las capacidades de los SIG (sistemas de información gerencial)

- Motivación de los empleados

\section{Productividad}

La productividad, se define como una de las variables claves para medir la eficacia y eficiencia en una organización, se define como un indicador que refleja el uso de los recursos y la relación que se tiene entre los recursos utilizados en la empresa en relación con los productos obtenidos, el indicador más utilizado hace referencia a la eficiencia de los recursos humanos en una organización, enfocada en la relación entre la cantidad de bienes y servicios producidos en relación a la cantidad de personal ocupado en una empresa (Colmenares, 2007).

La medición de la productividad debe contar con los siguientes elementos:

- Importancia en las decisiones tomadas

- Contar con precisión de la magnitud

- Debe permitir la toma de decisiones acertada por parte de la gerencia.

- Confiabilidad

- Proporcionalidad entre los costos de la empresa

La productividad se puede dividir en productividad parcial, total y marginal.

La productividad parcial, se relaciona con lo producido por un insumo o producto producido en relación a un recurso utilizado: (Carro Paz \& Gonzalez Gomez)

$$
\text { Productividad Parcial }=\frac{\text { Salida Total }}{\text { Una Entrada o Recurso }}
$$

La productividad total involucra a todos los recursos con los que cuenta la empresa, en relación a los productos o servicios entregados por la empresa:

$$
\text { Productividad Total }=\frac{\text { Bienes y Servicios Producidos }}{\text { Mano de Obra }+ \text { Capital }+ \text { Materias Primas }}
$$

La productividad marginal, se refiere a la cantidad de recursos empleados en la producción de una unidad adicional de la empresa.

La determinación de la productividad es esencial para los correctos planes en la empresa, que nace de una proyección clara de las metas y del acertado uso de los recursos, así como de los indicadores claves para el proceso de planificación empresarial.

La Mota, Iglesias, Zea. Modelo estratégico para optimizar la productividad de la empresa M\&D Catering 


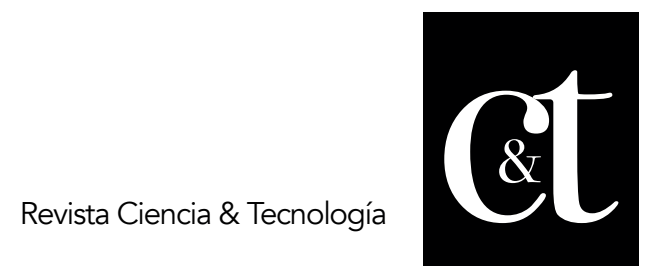

No. 30, 30 de abril de 2021

ISSN impreso: 1390 - 6321

ISSN online: 2661 - 6734

\section{Metodología}

El enfoque de la investigación es cualitativo debido a que mediante el análisis de la información secundaria se revisa cada elemento para determinar oportunidades de mejora en los procesos y actividades que se verán reflejadas en un diseño de planificación estratégica de la empresa (Hernández Sampieri, 2014).

El análisis de la cadena de valor es descriptivo utilizando como instrumentos de recolección de información la observación directa, la revisión de información documental y entrevistas a profundidad a los responsables del cumplimiento de los procesos primarios y secundarios de la cadena de valor de la empresa. Se procederá a realizar una planificación estratégica que incluye Objetivos Estratégicos, Objetivos Tácticos, Actividades. Para la cadena de valor el instrumento principal utilizado en la investigación es documental, revisando los procesos de la empresa relacionados a la cadena de valor para determinar oportunidades de mejora en los mismos además de realizar una observación directa para constatar la ejecución de dichos procesos y detallar hallazgos adicionales relacionados a la operación. Para complementar los elementos de la cadena de valor se realizará entrevistas a profundidad, con autorización debida, a los responsables de los procesos de la cadena de valor de la empresa estos son:

- $\quad$ Gerente General

- Gerente de Operaciones

- Responsable de Compras

- Responsable de Logística

Cada responsable aportará a la información recabada de la cadena de valor de acuerdo con sus actividades y responsabilidades en la empresa, a continuación, se detalla a que elementos de la cadena de valor se enfocará la entrevista a profundidad para cada responsable:

\section{Tabla 1. Enfoque para entrevistas a directivos de MYD Catering}

Cargo

Gerente

general
Elemento cadena de valor

Marketing y ventas

Servicio posventa

Desarrollo tecnológico

Recursos humanos

Infraestructura de la empresa

Gerente de

operaciones

Responsable

de compras

Responsable

de logística
Operaciones

Logística de entrada

Compras

Logística de salida

Fuente: elaboración propia 


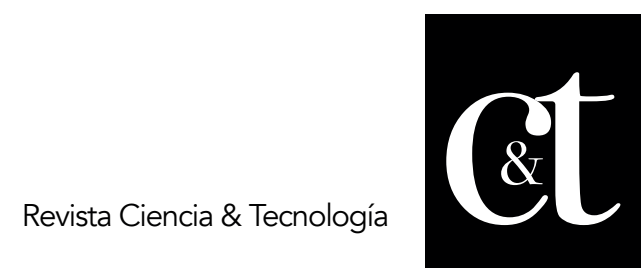

No. 30, 30 de abril de 2021

ISSN impreso: 1390 - 6321

ISSN online: 2661 - 6734

Para la Productividad se realizará un análisis documental de la información de la empresa registrada en los años 2018 y 2019, relacionada a los siguientes insumos:

- $\quad$ Listados de clientes.

- Organigrama de la empresa.

- $\quad$ Formatos y otros insumos utilizados por la empresa.

Con esta información documental se realiza un análisis cualitativo de los resultados obtenidos para la productividad de la empresa, y en función de las oportunidades de mejora detectadas en la cadena de valor, diseñar una planificación que busque mejorar los indicadores de productividad de la empresa.

\section{Resultados y discusión}

De la entrevista realizada al gerente general sobre los elementos de la cadena de valor a su cargo se destacan como oportunidades de mejora la automatización requerida de la operación para controlar las actividades primarias de la empresa, así como potenciar subsistemas de talento humano como el de evaluación y capacitación que permitan seguir marcando un crecimiento continuo para la empresa.

A continuación se presenta el cuadro con las principales oportunidades de mejora detectadas de las entrevistas realizadas a cada responsable.

La Mota, Iglesias, Zea. Modelo estratégico para optimizar la productividad de la empresa M\&D Catering 


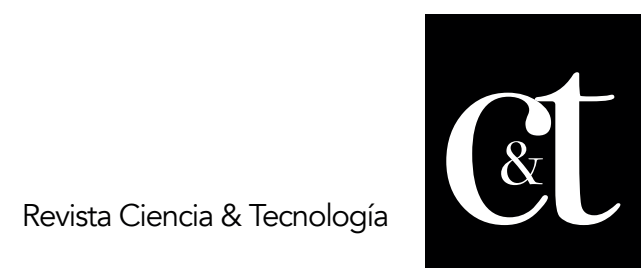

No. 30, 30 de abril de 2021

ISSN impreso: 1390 - 6321

ISSN online: 2661 - 6734

Tabla 2. Cuadro de oportunidades de mejora cadena de valor MYD CATERING

\begin{tabular}{|c|c|c|}
\hline Actividades & Elementos & Oportunidades de Mejora \\
\hline \multirow[t]{5}{*}{$\begin{array}{l}\text { Actividades } \\
\text { Primarias de } \\
\text { la Empresa }\end{array}$} & $\begin{array}{l}\text { Logística de } \\
\text { Entrada }\end{array}$ & $\begin{array}{l}\text { Implementar un sistema de } \\
\text { información para el control de } \\
\text { inventarios y materias primas. }\end{array}$ \\
\hline & Operaciones & $\begin{array}{l}\text { Certificar las operaciones y la } \\
\text { planta de producción en normas } \\
\text { BPM (buenas prácticas de } \\
\text { manufactura) }\end{array}$ \\
\hline & $\begin{array}{l}\text { Logística de } \\
\text { Salida }\end{array}$ & $\begin{array}{l}\text { Adquisición de vehículos y } \\
\text { desarrollo de procesos de } \\
\text { distribución y rutas criticas }\end{array}$ \\
\hline & $\begin{array}{l}\text { Marketing } \\
\text { Ventas }\end{array}$ & $\begin{array}{l}\text { Desarrollar un plan de } \\
\text { marketing, que determine las } \\
\text { acciones y estrategias a realizar }\end{array}$ \\
\hline & $\begin{array}{l}\text { Servicio } \\
\text { Posventa }\end{array}$ & $\begin{array}{l}\text { Generar incentivos de retención } \\
\text { a los clientes para mejorar su } \\
\text { satisfacción y fidelidad }\end{array}$ \\
\hline \multirow[t]{4}{*}{$\begin{array}{l}\text { Actividades } \\
\text { Secundarias } \\
\text { de la } \\
\text { Empresa }\end{array}$} & Compras & $\begin{array}{l}\text { Ampliar el proceso de } \\
\text { aprobación de menú y } \\
\text { requerimiento de compras de } \\
\text { semanal a mensual }\end{array}$ \\
\hline & $\begin{array}{l}\text { Desarrollo } \\
\text { Tecnológico }\end{array}$ & $\begin{array}{l}\text { Implementar un sistema de } \\
\text { información para el control } \\
\text { general de la operación en la } \\
\text { parte administrativa y de } \\
\text { producción de la empresa }\end{array}$ \\
\hline & $\begin{array}{l}\text { Recursos } \\
\text { Humanos }\end{array}$ & $\begin{array}{l}\text { Potenciar los subsistemas de } \\
\text { capacitación y evaluación de } \\
\text { desempeño para los } \\
\text { colaboradores, que articulen } \\
\text { beneficios e incentivos de } \\
\text { acuerdo con los resultados y a } \\
\text { su productividad }\end{array}$ \\
\hline & $\begin{array}{l}\text { Infraestructura } \\
\text { de la Empresa }\end{array}$ & $\begin{array}{l}\text { Realizar un plan de inversión } \\
\text { para adquisición de maquinarias } \\
\text { y otros recursos de capital para } \\
\text { la mejora de la productividad en } \\
\text { la empresa }\end{array}$ \\
\hline
\end{tabular}

Fuente: elaboración propia 


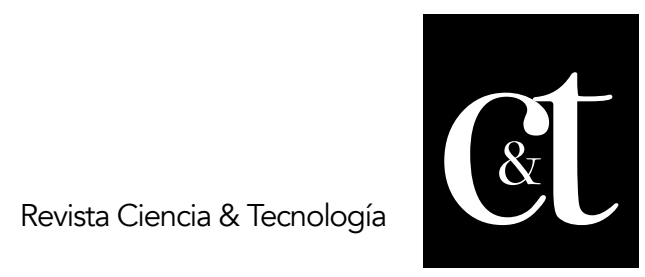

No. 30, 30 de abril de 2021

ISSN impreso: 1390 - 6321

ISSN online: 2661 - 6734

Para la observación directa hubo una guía de observación para cada macroproceso para verificar su cumplimiento, y se estableció la siguiente escala de medición en relación al cumplimiento de cada actividad detallada en el proceso. De la observación directa realizada a los 8 macroprocesos desarrollados por la empresa se obtiene el siguiente cuadro resumen donde se detalla los procesos que presentan una oportunidad de mejora.

\section{Tabla 3. Procesos de la empresa con oportunidades de mejora}

\begin{tabular}{|c|c|c|c|}
\hline Proceso & Calificación & Equivalencia & Observación \\
\hline \multirow{3}{*}{$\begin{array}{l}\text { Proceso de } \\
\text { Compras } \\
\text { (Efectivo) }\end{array}$} & \multirow[t]{3}{*}{3} & \multirow[t]{3}{*}{$\begin{array}{l}\text { Cumplimiento } \\
\text { Parcial }\end{array}$} & \multirow{3}{*}{$\begin{array}{l}\text { Se ha ido } \\
\text { eliminando el uso de } \\
\text { las compras en } \\
\text { efectivo, en su } \\
\text { mayoría en la } \\
\text { actualidad } \\
\text { manejan a crédito }\end{array}$} \\
\hline & & & \\
\hline & & & \\
\hline Proceso de & 3 & Cumplimiento & \multirow[b]{2}{*}{$\begin{array}{l}\text { Se evidencia } \\
\text { demora en el } \\
\text { proceso de registro } \\
\text { de compras en el } \\
\text { sistema y en la } \\
\text { contabilización de } \\
\text { registros }\end{array}$} \\
\hline $\begin{array}{l}\text { Compras } \\
\text { (Crédito) }\end{array}$ & & Parcial & \\
\hline Ventas & 3 & $\begin{array}{l}\text { Cumplimiento } \\
\text { Parcial }\end{array}$ & $\begin{array}{l}\text { No se evidencia } \\
\text { facturación } \\
\text { utilizando el sistema } \\
\text { de acuerdo a platos } \\
\text { o ítems generados } \\
\text { desde las ordenes } \\
\text { de producción }\end{array}$ \\
\hline $\begin{array}{l}\text { Supervisión } \\
\text { del registro }\end{array}$ & 2 & $\begin{array}{l}\text { Cumplimiento } \\
\text { insuficiente }\end{array}$ & $\begin{array}{l}\text { No se evidencia el } \\
\text { uso del sistema para }\end{array}$ \\
\hline & & & los registros \\
\hline operaciones & & & 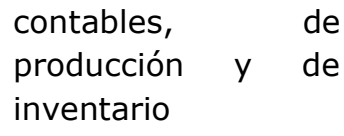 \\
\hline
\end{tabular}

Fuente: elaboración propia

\section{Comparación de la productividad mensual por trabajador}

Se evidencia que la empresa ha mejorado sus niveles de productividad, contando con menos personal en la planta de producción y teniendo mayores niveles individuales de productividad en el servicio. Para analizar la satisfacción de clientes se ha considerado calcular el índice de retención de clientes para el año 2018 y el año 2019 de la siguiente forma: 


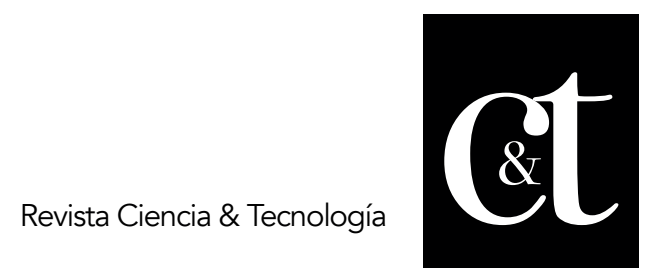

No. 30, 30 de abril de 2021

ISSN impreso: 1390 - 6321

ISSN online: 2661 - 6734

Índice de retención de clientes $=\frac{(\# C l i e n t e s \text { Final Período }-\# \text { Clientes Nuevos })}{\text { \#Clientes al inicio del período }} * 100$

\section{Tabla 4. Índice de retención de clientes}

\begin{tabular}{l|llll}
\multirow{2}{*}{ Año } & $\begin{array}{l}\text { Clientes } \\
\text { Inicio } \\
\text { Periodo }\end{array}$ & $\begin{array}{l}\text { Clientes } \\
\text { Final } \\
\text { Periodo }\end{array}$ & $\begin{array}{l}\text { Clientes } \\
\text { Nuevos } \\
\text { Periodo }\end{array}$ & $\begin{array}{l}\text { Índice de } \\
\text { Retención }\end{array}$ \\
\hline 2018 & 7 & 4 & 0 & $57 \%$ \\
2019 & 4 & 10 & 6 & $100 \%$
\end{tabular}

Elaborado por: Gustavo La Mota Terranova

De los resultados obtenidos se determina como propuesta principal el cuadro de mando integral para la empresa, el cual tiene como fuente las oportunidades de mejora obtenidas del análisis de la cadena de valor.

Tabla 5: Cuadro resumen balanced scorecard M\&D Catering

\begin{tabular}{|c|c|c|}
\hline Perspectiva & Cod & Objetivos Estratégicos Generales \\
\hline $\begin{array}{l}\text { Perspectiva } \\
\text { de Innovación } \\
\text { y Aprendizaje }\end{array}$ & I1 & $\begin{array}{l}\text { Capacitar al personal de la planta en buenas prácticas de } \\
\text { manufactura }\end{array}$ \\
\hline $\begin{array}{l}\text { Perspectiva } \\
\text { de los } \\
\text { Procesos } \\
\text { Internos }\end{array}$ & P1 & Fortalecer el control de la operación \\
\hline $\begin{array}{l}\text { Perspectiva } \\
\text { de los } \\
\text { Clientes }\end{array}$ & $\mathrm{C} 1$ & Captar nuevos clientes empresariales y corporativos \\
\hline $\begin{array}{l}\text { Perspectiva } \\
\text { Financiera }\end{array}$ & $\mathrm{F} 1$ & $\begin{array}{l}\text { Realizar las inversiones y adecuaciones requeridas en la } \\
\text { infraestructura }\end{array}$ \\
\hline
\end{tabular}

Fuente: elaboración propia

Del análisis interno y externo de los factores de la empresa, la verificación del cumplimiento de los procesos y los resultados de las entrevistas realizadas a los responsables de la empresa de acuerdo a la cadena de valor se propone un modelo estratégico para la empresa MYD Catering, el cual se basa en optimizar la productividad de la empresa y la retención de sus clientes para el largo plazo.

El presente modelo estratégico toma como base el modelo de cuadro de mando integral (balanced scorecard) para definir los objetivos y actividades de la empresa en las distintas perspectivas y los indicadores clave para medir la consecución de esos objetivos en la empresa.

Con los elementos del balanced scorecard ubicados se puede determinar un mapa estratégico de la empresa que permite seguir el curso de acción de la compañía para optimizar su productividad en función a la cadena de valor. 


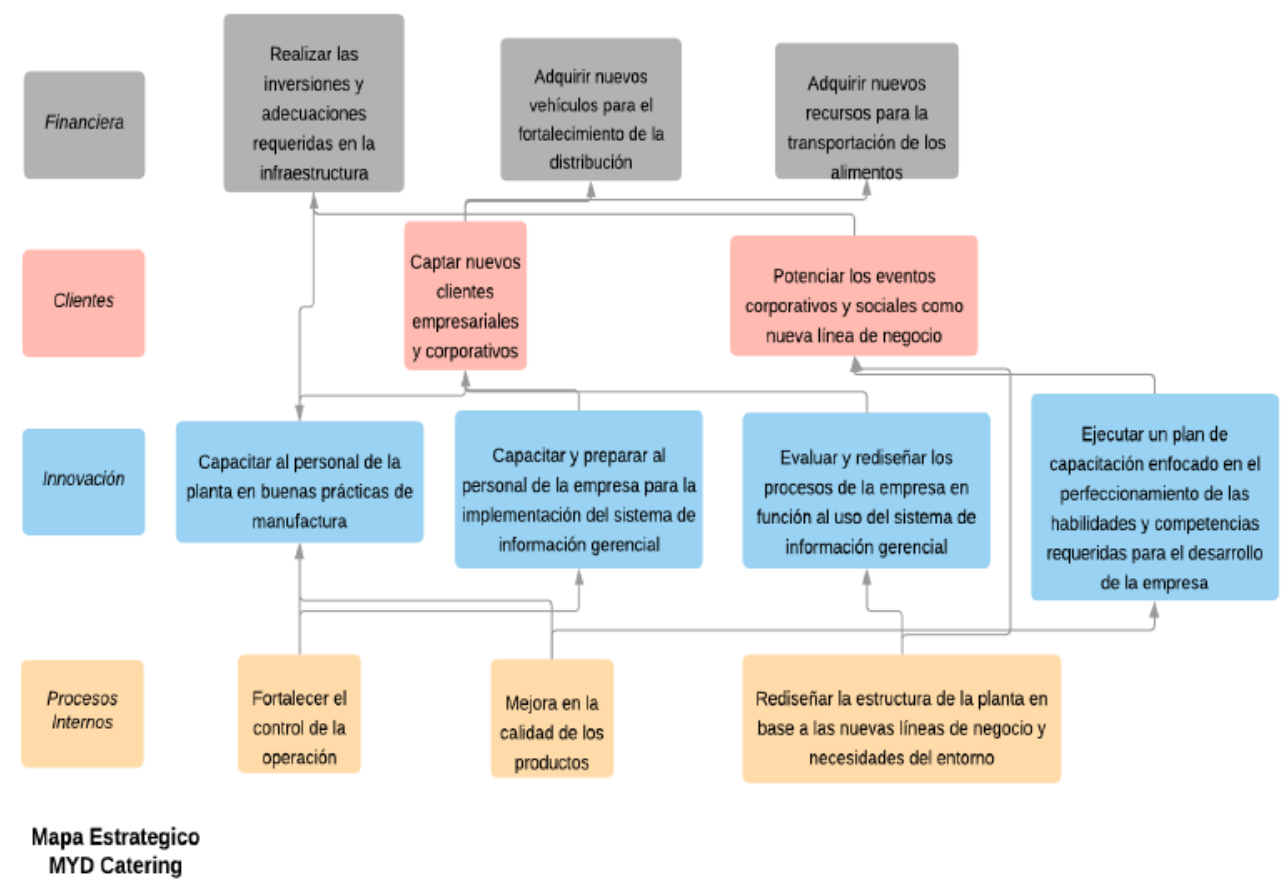

Figura 1: Mapa estratégico de MYD CATERING

Fuente: elaboración propia

\section{Conclusiones}

Luego de una verificación y análisis de los procesos y los elementos de la cadena de valor de la empresa se evidencia que las prácticas y procesos realizados por la empresa MYD Catering han optimizado y mejorado los indicadores de productividad y retención de clientes de las empresas, y que a través de la propuesta estos se verán optimizados para continuar ese crecimiento continuo en la organización.

Del análisis de la cadena de valor se constató que al menos cada elemento de la cadena podría presentar una oportunidad de mejora resaltada por los responsables de las actividades primarias y de apoyo de la empresa, muchos departamentos convergieron en la necesidad de implementar un sistema de información gerencial para el control operativo de la planta como una alternativa esencial en el desarrollo de la impresa, misma que claramente se ve evidenciada en la propuesta de planificación estratégica diseñada para la empresa.

La planificación estratégica propuesta de la empresa se presenta como resultado de un análisis de las oportunidades de mejora obtenidas del análisis de la cadena de valor y de la verificación y el cumplimiento de procesos de la empresa, el modelo estratégico utilizando el Balanced Scorecard detalla los objetivos y actividades que deben realizarse a partir del 2020 para optimizar la productividad de la empresa MYD Catering. 


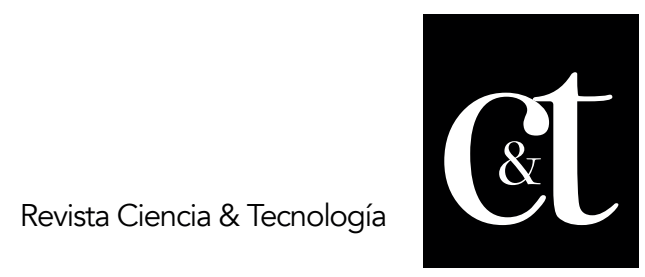

No. 30, 30 de abril de 2021

ISSN impreso: 1390 - 6321

ISSN online: 2661 - 6734

Como complemento al estudio de la cadena de valor para optimizar la productividad de la empresa, se recomienda enfocar un posterior estudio hacia el análisis externo de factores.

Para la implementación del sistema de información gerencial, se debe analizar en detalle las funciones específicas operativas que este debe cubrir para que la empresa o software contratado realmente coadyuve a una mejora en el control de la operación como objetivo para optimizar la productividad.

\section{Referencias}

Carrión Erazo, L. E., Zula Cujano, J. A., \& Castillo Guilcapi, L. Y. (2016). Pequeñas y Medianas Empresas Hacia un Nuevo modelo de Gestión en la industria del catering en el Ecuador. Observatorio de la Economía Latinoamericana.

Carro Paz, R., \& Gonzalez Gomez, D. (2012). Productividad y Competitividad. Argentina: Universidad Nacional Mar del Plata.

Castillo, L., Zula, J., \& Carrión, L. (2016). Estudio mercado del servicio de catering para las empresas CONSEP $Y$ CONQUITO EN ECUADOR. Observatorio de la economía latinoamericana.

Colmenares, O. (2007). Medición de la productividad empresarial.

Hernández Sampieri, R., Fernández Collado, C., \& Baptista Lucio, P. (2014). Metodología de la investigación 6ta edición . Colombia: Mc Graw Hill.

Iglesias Mora, P. (2015). Diseño de un modelo de gestión utilizando la planificación estratégica como herramienta clave para lograr la competitividad y la permanencia de las pequeñas y medianas empresas familiares en el mercado global, caso Guayaquil-Ecuador. UNMSM. LimaPerú: Tesis Doctoral.

Iglesias, D. H. (2002). Cadena de Valor como estrategia en el sector alimentario. INTA.

Kaplan, R., \& Norton, D. (2000). Cuadro de Mando Integral. Ediciones Gestión. La Mota, G. (2019). Modelo estratégico para optimizar la productividad de la empresa MyD Catering aplicando la cadena de valor. Tesis de maestría. UTEG.

Olmedo, F., Olmedo, F., \& Plazaola, N. (s/f). Cadena de Valor. Estrategia Magazine Edición 19.

Palacios Acero, L. (s.f.). Dirección Estratégica. ECOE Ediciones.

Porter, M. (1982). Estrategia Competitiva. México: Compañía Editorial S.a de C.v.

Quintero, J., \& Sánchez, J. (2006). La cadena de valor: Una herramienta de pensamiento estratégico. Telos.

Romo Morales, G., \& Márquez de León, E. (2014). Gestión estratégica vs Análisis estratégico. Una discusion coceptual a partir del estudio de caso de una institucion de educacion superior. Pensamiento y gestión.

Saavedra García, M., \& Espíndola Armenta, G. (2016). El uso de la planeación financiera en las PYME de TI de méxico. Ciencias Administrativas.

Sablon Cossío , N., Medina León, A., Acevedo Suarez, J. A., Acevedo Urquiaga, A. J., \& López Joy, T. (2013). Consideraciones sobre la planificación de productos alimenticios en una cadena de suministro comercial. Revista Ingeniería industrial. 


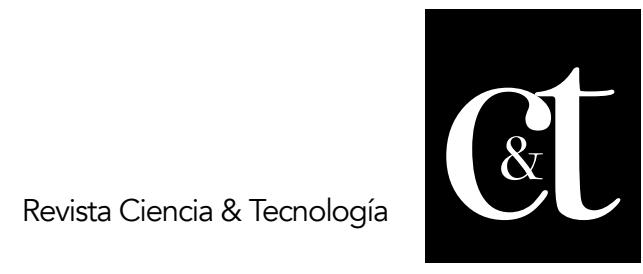

No. 30, 30 de abril de 2021

ISSN impreso: 1390 - 6321

ISSN online: 2661 - 6734

Sánchez Quintero, J. (2003). Estrategia Integral para PYMES Innovadoras. Revista Escuela de Administración y Negocios. núm. 47, enero-abril.

Sánchez Vásquez, J., Vélez Elorza, M., \& Araujo Pinzón, P. (2016). Balanced Scorecard para emprendedores desde el modelo canvas al cuadro de mando integral. Facultad de Ciencias Económicas.

La Mota, Iglesias, Zea. Modelo estratégico para optimizar la productividad de la empresa M\&D Catering 